\title{
Investigation and characterization of human gut phageome in advanced liver cirrhosis of defined etiologies
}

\author{
Mohadeseh Naseri ${ }^{1}$, Fahimeh Palizban², Abbas Yadegar ${ }^{3}$, Mohsen Khodarahmi $^{1,4}$, Hamid Asadzadeh Aghdaei $^{5}$, \\ Hamidreza Houri ${ }^{3^{*}}$ (1) and Javad Zahiri $i^{*}$
}

\begin{abstract}
Background: Liver cirrhosis is a major public health problem, accounting for high rates of morbidity and mortality worldwide. The cirrhosis etiology is a broad and essential step in planning a treatment strategy. Many recent studies have documented that gut microbiome alterations play a vital role in the development and progression of cirrhosis and its complications. Nevertheless, there is insufficient data on the correlation between liver cirrhosis and gut phageome alterations in patients with advanced liver diseases. This study aimed to analyze the taxonomic structure and functional attributes of the gut phageome in six different etiologies of advanced liver cirrhosis.
\end{abstract}

Methods: We first retrieved metagenomic sequencing data from three datasets of fecal samples taken from cirrhotic patients with various etiologies. Subsequently, several bioinformatics pipelines were used to analyze bacteriophage composition and determine their functionality.

Results: A gene catalog of 479,425 non-redundant genes was developed as a reference to measure gene prevalence. The results of the analysis revealed a few significant differences among the cohorts at the phage species level. However, the alternations were more evident as there were more in-depth analyses of the functional resolution of the gut phageome.

Conclusions: Our findings suggest that the functional analysis of the gut phageome would provide meaningful markers to predict the progression of liver cirrhosis and facilitate the development of novel treatment approaches.

Keywords: Liver cirrhosis, Phageome, Metagenomics, Nonalcoholic fatty liver disease

\section{Introduction}

The human gut microbiome comprises an extremely diverse and complex community of microorganisms and is a highly dynamic ecosystem affecting the host health status and accelerating the disease progression [1]. There

\footnotetext{
*Correspondence: hr.houri@sbmu.ac.ir; jzahiri@health.ucsd.edu

${ }^{3}$ Foodborne and Waterborne Diseases Research Center, Research Institute for Gastroenterology and Liver Diseases, Shahid Beheshti University of Medical Sciences, Shahid Arabi Ave., Yemen St., Velenjak, Tehran, Iran

${ }^{6}$ Department of Neuroscience, University of California, San Diego, 9500 Gilman Drive, La Jolla, CA 92093-0662, USA

Full list of author information is available at the end of the article
}

has been an increased interest in understanding the role of the gut microbiome in developing metabolic disorders, with some studies sparing efforts to elucidate the functional significance of the microbiome in the progression of liver diseases [2,3]. Nowadays, the metagenomics analysis of the human-associated microbiome provides a rich set of microbial features for prediction and biomarker discovery in the context of human diseases and health conditions [4]. Among these diseases, liver disorders annually account for about 2 million deaths worldwide, in which more than 1 million cases are diagnosed with liver cirrhosis complications [5]. Liver original author(s) and the source, provide a link to the Creative Commons licence, and indicate if changes were made. The images or other third party material in this article are included in the article's Creative Commons licence, unless indicated otherwise in a credit line to the material. If material is not included in the article's Creative Commons licence and your intended use is not permitted by statutory regulation or exceeds the permitted use, you will need to obtain permission directly from the copyright holder. To view a copy of this licence, visit http://creativecommons.org/licenses/by/4.0/. The Creative Commons Public Domain Dedication waiver (http://creativeco mmons.org/publicdomain/zero/1.0/) applies to the data made available in this article, unless otherwise stated in a credit line to the data. 
cirrhosis is an irreversible end result of a long-lasting clinical course of several chronic liver diseases, which are commonly a consequence of long-term alcohol abuse, infection with hepatitis viruses, nonalcoholic fatty liver disease, etc. [6, 7]. More importantly, the human gut microbiome seems to play a vital role in the development and progression of cirrhosis $[8,9]$. For example, Loomba et al. conducted a study to identify the gut microbiome signatures of advanced fibrosis in patients with Nonalcoholic Fatty Liver Disease (NAFLD) [10]. Similarly, Zhao et al. characterized the gut microbiota composition and the functional annotations of Chinese children and adolescents with NAFLD [11].

To date, most microbiome studies have focused on the bacterial composition of the human gut, and less attention has been paid to the detailed analysis of the whole community of viruses and bacteriophages, henceforth called virome and phageome, respectively [12, 13]. More recently, the contribution of phage populations to the gut microbiome ecology and their effects on human health and diseases has been to be deciphered. Emerging data indicate that gut-associated phages can play a significant role in gastrointestinal physiology by regulating bacterial density, maintaining biodiversity, and controlling network interactions among the gut bacterial communities [14-16].

Previously, healthy individuals were assumed to have a stable gut phageome, which predominantly constituted of non-enveloped double-stranded DNA (dsDNA) Caudovirales or single-stranded DNA (ssDNA) Microviridae, as well as ssDNA filamentous phages, i.e., Inoviridae, reproduced by chronic infection without killing their host [17-19]. Interestingly, any compositional changes in the gut phageome could be associated with a variety of gut-related and systemic disorders such as inflammatory bowel diseases (IBD) [14], AIDS [20], and even malnutrition [21]. For example, an in-depth metagenomics analysis revealed a significant increase in the number of enteric Caudovirales phages in patients with Crohn's disease (CD) and ulcerative colitis (UC) [14]. Ma et al. carried out the first study exploring the large and diverse communities of gut phages in type II diabetes and declared the significance of the phageome in type II diabetes [22]. However, these investigations address a key obstacle: there is currently no single recognized database of annotated virus genome sequences, and de novo prediction of virus sequences from metagenomic assemblies has remained a remarkable challenge.

It is worth mentioning that little attention has been paid to detecting the role of gut phageome in liver diseases; hence, future studies need to delve into the characteristics of the gut phageome in various liver disorders. Moreover, access to a reference gene catalog is inevitable to deeply investigate microbial environments such as the human gut. Accordingly, there have been several attempts to construct specific human microbiome gene catalogs containing several types of microorganisms, mainly bacterial species [23]. In this regard, a better understanding of the phage content of the human gut depends on access to a comprehensive gene catalog of the gut phageome. In the present study, we adopted three bioinformatic strategies to identify the large scaffolds of phage origin to investigate the structural and functional composition of gut phageome in several etiologies of liver diseases. This is the first study to correlate the gut phageome with liver cirrhosis to provide valuable insights into the design of novel phage-related markers in monitoring liver disease progression and its treatment.

\section{Methods}

Input data characteristics and collection procedure

In this study, three previously published metagenomic datasets were employed for the analyses. In this regard, three metagenomic datasets were obtained from the National Center for Biotechnology Information's (NCBI's) Sequence Read Archive (SRA) to investigate the gut phageome of cirrhotic patients from structural and functional aspects deeply. The total number of participants with liver cirrhosis in these three studies was 139, of whom 98 samples belonged to the Chinese population (PRJEB6337). The dataset of the Chinese population was categorized as alcohol-induced cirrhosis (Chinese ALC, $n=10$ ), hepatitis B virus-related cirrhosis (Chinese $H B V, n=30)$, a combination of hepatitis $B$ virus and alcohol-related cirrhosis (Chinese ALC-HBV) $(n=24)$, and other etiologies (e.g., hepatitis $\mathrm{C}, \mathrm{E}$, and D virus, etc.; $\mathrm{n}=34$ ) [24]. The metagenomic dataset, which was reported by Tyakht et al. and obtained from 27 Russian patients with alcohol-related cirrhosis, was included as the Russian ALC cohort (PRJNA373901) [25]. Finally, 14 nonalcoholic fatty liver disease (NAFLD) patients with advanced-stage fibrosis, who were evaluated by Loomba et al., were included as the NAFLD cohort (PRJEB18041) [10]. The quality of the sequencing reads was verified using FastQC software before further analysis [26]. Furthermore, the host residual genome content was checked in gut metagenomic data by aligning the reads to human reference genome hg38 using Bowtie2 [27]. Finally, due to the batch effects of different groups, the remove batch effect function was considered from the limma package [28].

\section{Phage catalog construction}

The main objective of this study was to construct the gut phage catalog facilitating the structural and functional analysis for the phageome of liver cirrhosis. To 
this end, the proposed approach encompassed three different strategies, as illustrated in Fig. 1. Furthermore, the high-quality metagenomic reads were analyzed by the FastViromeExplorer tool [29] to determine the gut phage composition in various etiologies of cirrhosis. The genetic sequences of known strains were identified by FastViromeExplorer and retrieved from the NCBI Refseq. Furthermore, we spared our efforts to determine all spacers from the 139 assembled metagenomic samples to improve the generality of our catalog. In this regard, metagenomic assembly was performed by MEGAHIT [30], and CRISPRFinder [31] was then used to predict all potential spacers in a unified assembled file. These spacers were mapped to a locally developed database of all available phages, which was made by integrating the phage sequences of NCBI and phage database (https:// phagesdb.org/). We also prepared a comprehensive database encompassing the genome of all known phages from distinct resources. Accordingly, 50,740 and 2816 phage genomes were obtained from NCBI and phagesDB, respectively. This database would provide the grounds for the more accurate and efficient investigation and identification of gut phageome. Further, MetaGeneMark with default flags was used to generate the nucleic acid and amino acid sequences related to the predicted genes from the assembled file. Moreover, the protein sequences of the predicted genes were mapped to the previously mentioned local phage database by the DIAMOND alignment tool [32]. Finally, all genes identified by the three strategies were integrated into one single gene catalog, and CD-HIT [33] was then applied to remove redundant genes (with a 90\% similarity threshold).

\section{Phage community analysis and taxonomic profiling}

Before any phage community analysis, several filtrations were applied on phage species, genus, and family resolutions to eliminate the wrong and pseudo correlations. Accordingly, a taxon with $<0.001$ prevalence in more than five samples was removed. The Vegans [34] and Phyloseq R [35] packages were used to measure the dissimilarity and alpha diversity (i.e., ACE, Shannon, and Simpson indices), respectively. The differences among the mentioned variables in several phenotypes were detected by the ANOVA test. Further statistical analysis and visualization steps were conducted using ggplot $2 \mathrm{R}$ packages [36].

\section{Functional analysis of human gut phageome}

We developed a phage gene catalog to identify the putative biochemical interaction networks between the gut phageome and microbiome. Accordingly, the raw metagenomic samples were mapped to the gene catalog by the MOSAIK aligner [37], and samtools idxstats [38] were then used to determine the frequency of each covered gene in each sample. Differentially prevalent genes in each cohort were normalized and extracted by the edgeR package [26]. In the next step, we investigated the gene sets specific for each etiology based on their

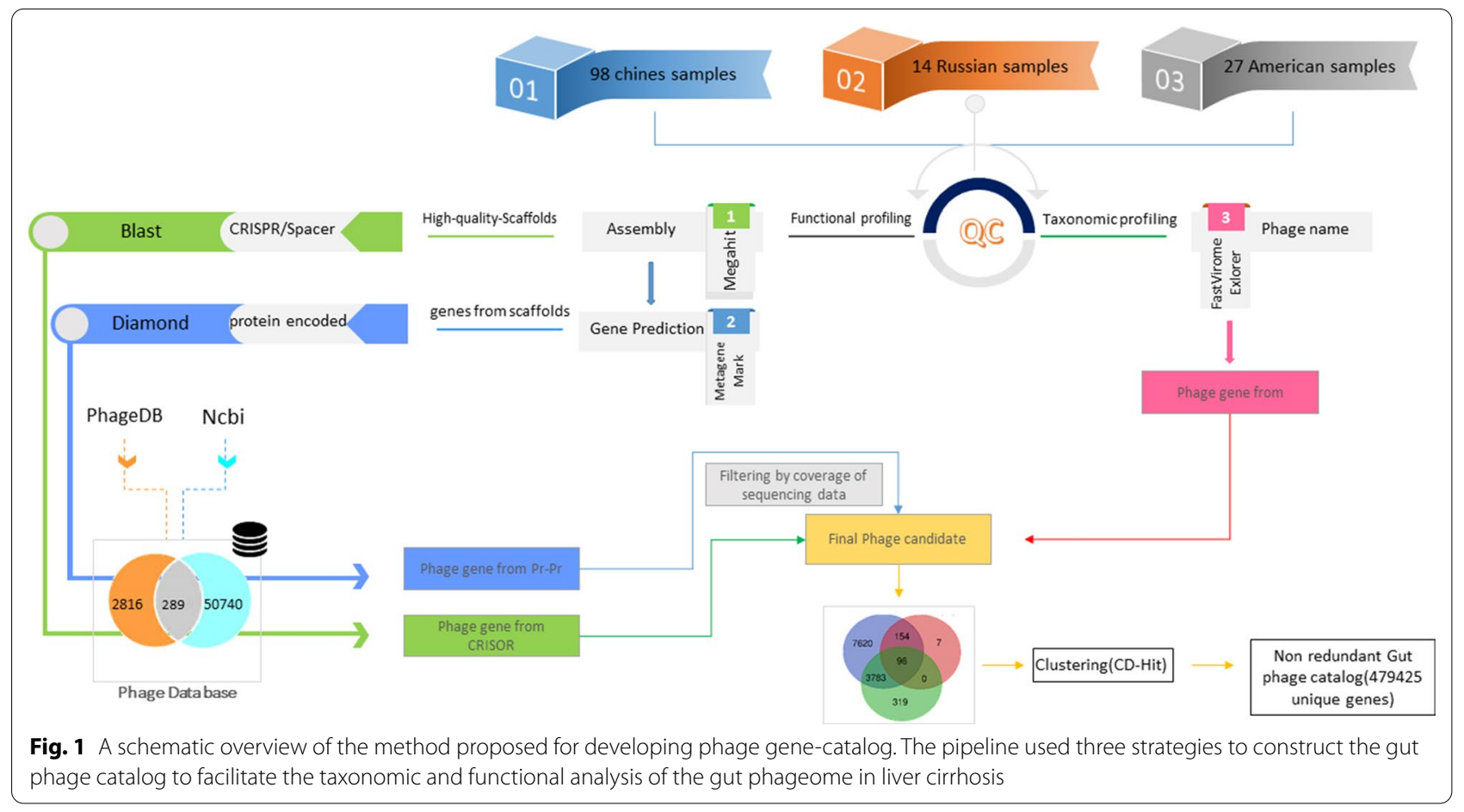


abundance and statistical test analysis. Accordingly, we found the specific and significantly related gene sets for each etiology by using edgeR based on the fold change and adjusted $p$-value. Then common genes among different groups were omitted and the rest of them were extracted and assigned as an etiology-specific gene set. Afterward, the GhostKOALA tool [39] was applied to detect the KEGG orthology of the genes.

\section{Gene annotation of the differentially prevalent genes}

In addition to the $\mathrm{KO}$ analysis, the functional annotation of the genes extracted by edgeR was performed by comparing the genes in different databases, including the GenBank, COG [40], eggNOG 4.5 [41], and Pfam [42] using RPS-BLAST [43] with an e-value cutoff of $1 \mathrm{e}-10$. The annotation results were visualized by FuncTree2 [44].

\section{Results}

\section{Phage gene catalog construction and core phageome} in liver cirrhosis

Before applying the structural and functional analysis, a phage gene catalog of distinct etiologies of liver cirrhosis was developed in this study. Using three bioinformatic strategies, we recognized large phage scaffolds from the assembled scaffolds of the metagenomic data, including 479,425 non-redundant genes (the phage scaffolds were searched by (1) spacers of CRISPRs, (2) the known scaffolds were identified by FastViromeExplorer, and (3) the scaffold encoded proteins homologous to the proteins from a locally database of phages). As shown in Fig. 2A, the highest proportion of genes (35\%) in the catalog belonged to unclassified phages, followed by the phage genes of the family Siphoviridae (30\%). The unclassified phage scaffolds of the catalog could be an appropriate source for further analysis to detect and propose novel phage genes and strains. The large phage scaffolds ranged from 18 to $257 \mathrm{~kb}$ in length, consistent with the long-range of phage genomes in the family Siphoviridae (Fig. 2B).

This study investigated the core phageome of distinct etiologies of liver cirrhosis using three bioinformatic strategies. Although most of the detected large phage scaffolds were unique to each sample, 21 phage species were found to be common among all cohorts and defined as the core phageome in cirrhosis, as existed in more than two-thirds of the fecal samples of cirrhotic patients (Fig. 3). Among the 21 phages, uncultured crAssphage (NC_024711.1) was the most prevalent strain in all cohorts.

\section{Enteric phage diversity and gut phageome composition in various cirrhotic groups}

To find any specific alternation in the gut phageome among cirrhotic patients with an individual etiology, we first tried to identify the phage diversity in the cirrhotic cohorts with various etiologies. According to the FastViromeExplorer, 258 phage strains were unique to only one etiology. Increasing or decreasing $\alpha$-diversity in phage

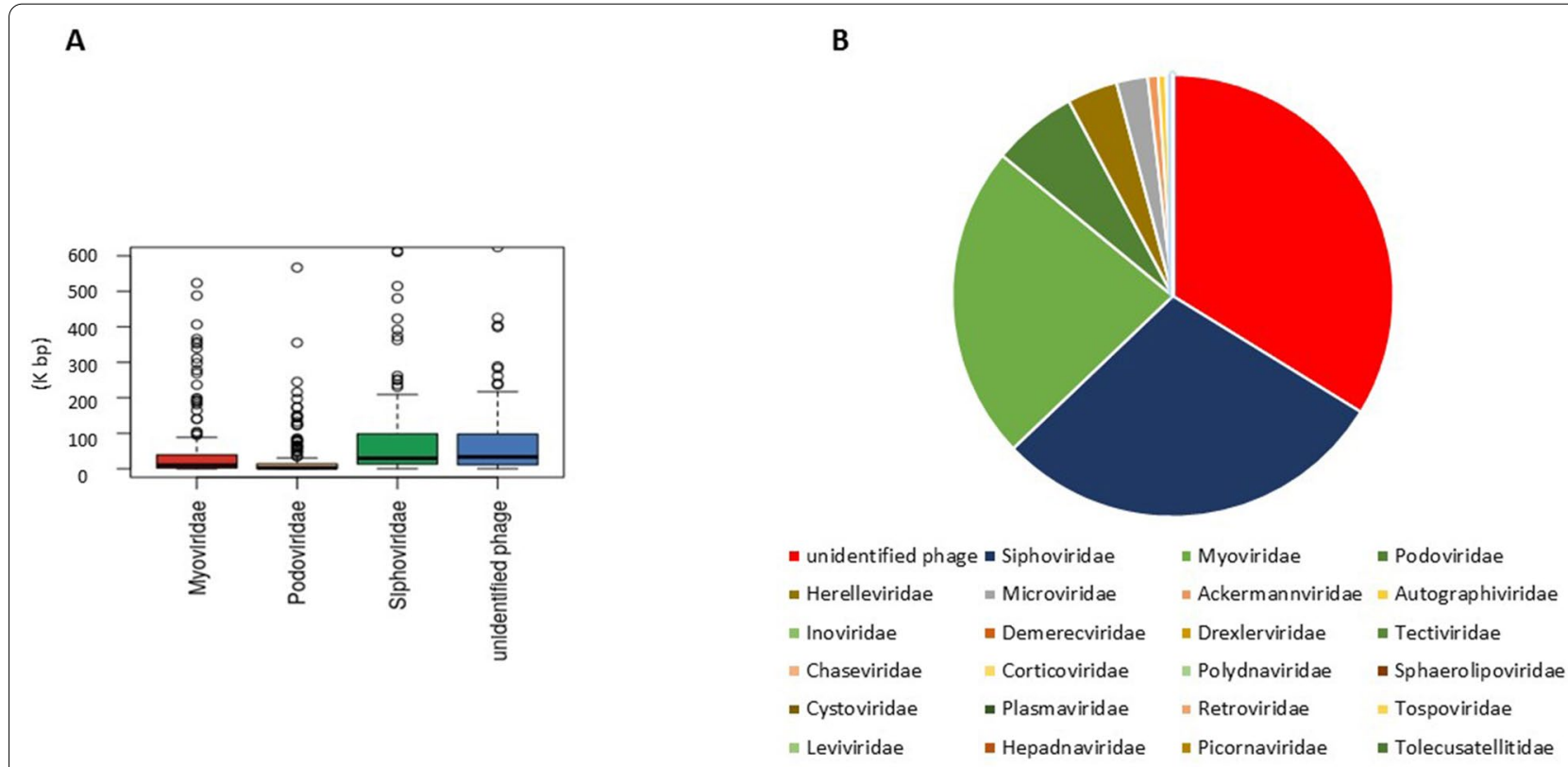

Fig. 2 A Length range of the genomes mapped in different phage families, B The most prevalent phage families in constructed phage gene-catalog 


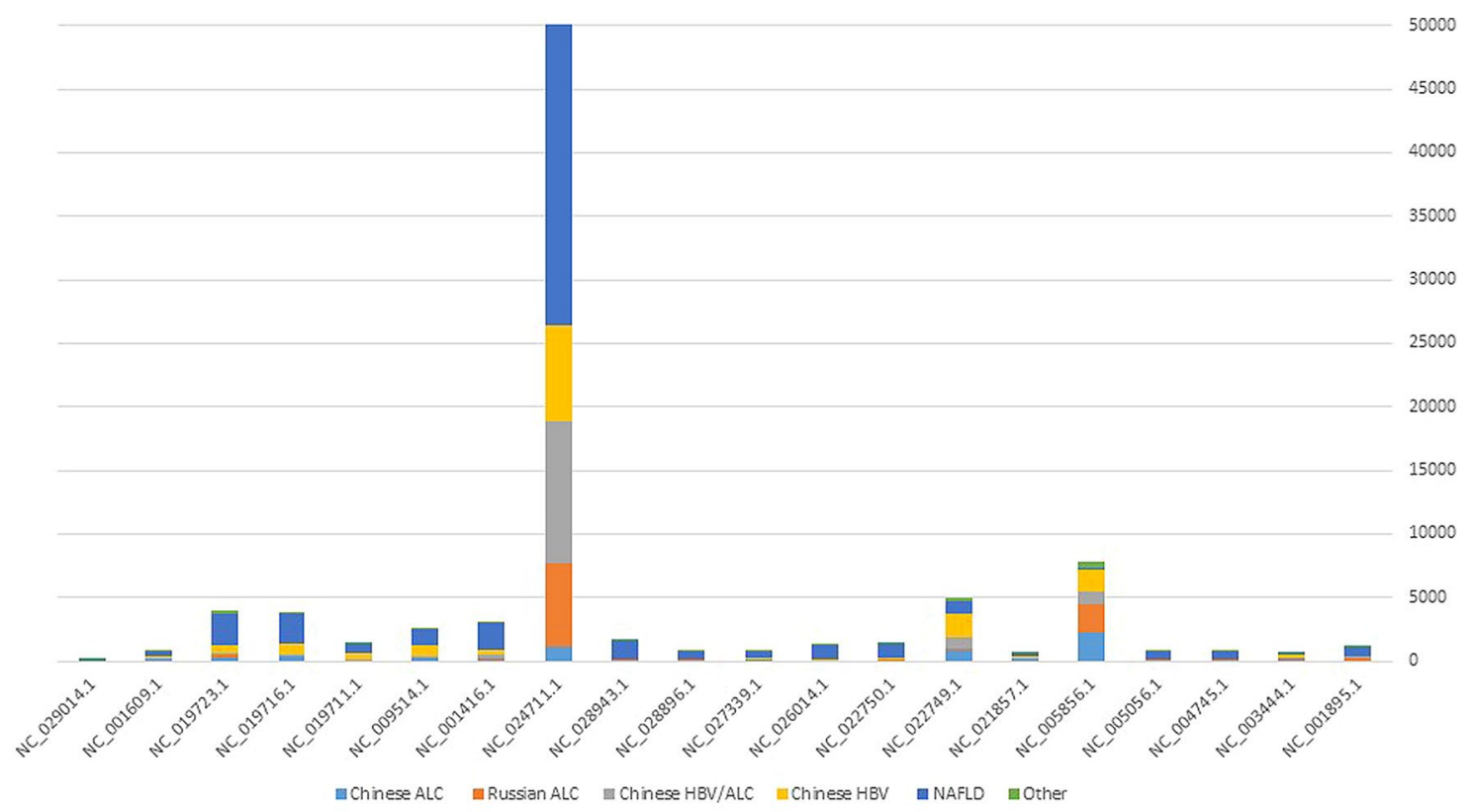

Fig. 3 Frequency distribution of each phage belonging to core phageome among different etiologies of liver disorders. Uncultured crAssphage (NC_024711.1) was the most prevalent phage in the core phageome of cirrhotic patients

composition among the different cirrhotic groups was examined using the Simpson index. Phage richness was not statistically different among the cirrhotic cohorts; however, there was a slight nonsignificant tendency for reduction of $\alpha$-diversity in the Chinese ALC cohort by reduced Simpson index (Fig. 4).

The relative frequency analysis at the family level (with 14 different families) revealed Siphoviridae as the most prevalent family in all cirrhotic cohorts, followed by Podoviridae and Myoviridae (Fig. 5). Individually, the analyses indicated that the Retroviridae family had been eliminated in the Chinese ALC and NAFLD groups. Moreover, post hoc pairwise analysis indicated that Introviridae showed a higher relative prevalence in the NAFLD group compared to the other groups. An analysis of the relative prevalence of phage taxa among Chinese

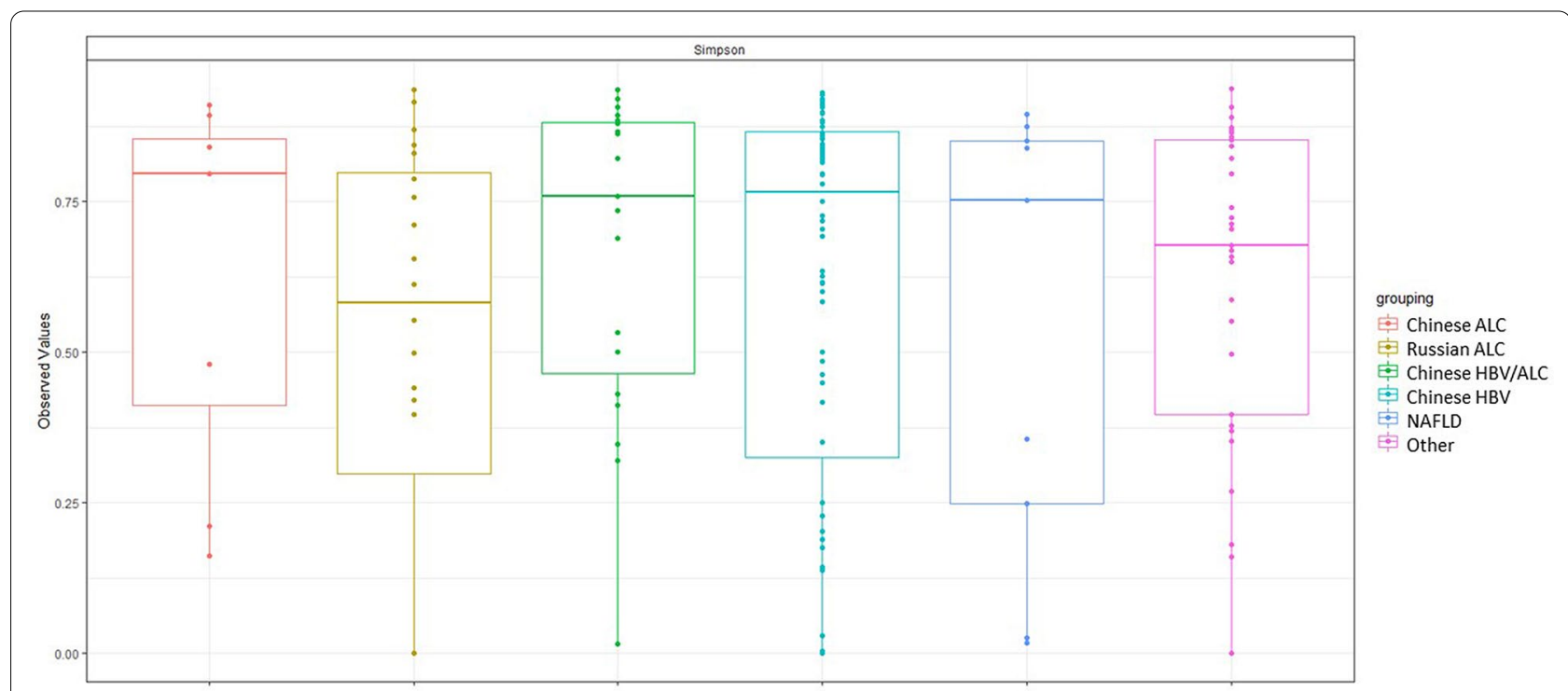

Fig. 4 Bacteriophage population a-diversity (Simpson index) in cirrhotic patients with various etiologies 


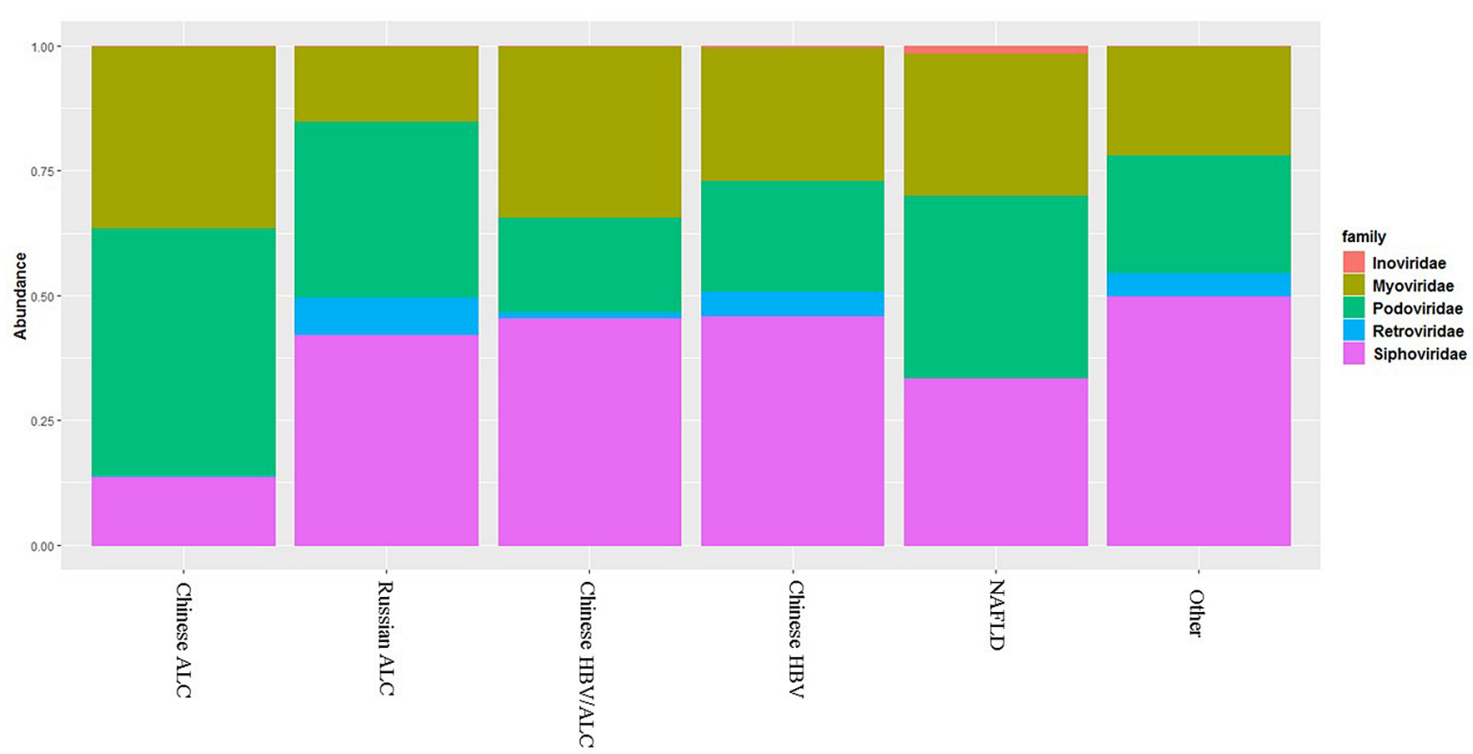

Fig. 5 Comparing the relative frequency of dominant bacteriophage families in different cirrhosis cohorts

cohorts revealed no significant difference among them at the family level regarding the etiology of cirrhosis, implying that the gut phageome could be strongly affected by geographical, racial, social, and dietary factors.

In the next step, we assessed phage species detectable only in one cirrhotic cohort to identify the presence or the absence of individual phage species and find a phageome signature in each etiology. Our analysis revealed that the Pseudomonas virus Pf1 phage, which belonged to the Inoviridae family, was only detected in the NAFLD group. Remarkably, Leuconostoc and Lactococcus phages belonging to the family Siphoviridae were identified as the individualized phage strains in the Russian ALC group. Interestingly, the results of the present study also indicated that most of the phages in the Chines cohorts were specific to the Enterobacteriaceae hosts, particularly Klebsiella spp. Figure 6 illustrates the individual phage richness in each cirrhotic group exhaustively.

To investigate phage compositional differences between Chines cirrhotic patients with different etiologies (i.e., Chines ALC and Chines HBV), we analyzed the prevalence of phage species unique to each group. According to the findings, four phages of the family Siphoviridae with completely different prevalence rates were identified in the Chines HBV and Chines ALC cohorts. These phages belonged to the unclassified Siphoviridae group (Enterobacteria phage cdtI and Enterobacteria phage mEp460) and the genus Lambdavirus (Escherichia virus Lambda and Escherichia phage HK629). As presented in Fig. 7, all four phages had higher frequencies in the Chinese HBV group. Moreover, we compared the NAFLD and ALC (i.e., Chinese ALC and Russian ALC) groups regarding the composition of the phage community at the species level. Notably, the uncultured phage crAssphage from the Podoviridae family was significantly higher in the NAFLD group compared to the ALC cohorts.

\section{Functional annotation of the enriched gene sets}

We also aimed to investigate functional markers as suitable predictive factors for each case of liver cirrhosis with different etiologies. Accordingly, by mapping each of the 139 samples to the constructed phage gene catalog, the prevalence of each gene in each sample was measured and then normalized based on the TMM normalization method of the edgeR package. Additional files 1, 2, 3, 4 and 5 present the frequencies of specific genes for each liver cirrhosis etiology.

Furthermore, the KEGG orthology (KO) analysis was carried out to deeply understand the exact biological mechanisms in the phage community of the human gut in different liver cirrhosis. To this end, the specific mapped genes in each phenotype were firstly extracted, and GkostKOALA was then used to determine the KOs of each phenotype. According to our analysis, K00986 and K06905 were the most common in all cirrhotic groups, which were related to RNA-directed DNA polymerase and uncharacterized protein, respectively. Comparatively, the Russian ALC and NAFLD phenotypes had the most distinct KOs with the frequency of 87 and 36 numbers, respectively (Additional file 6). More importantly, regarding KOs, the samples belonging to the same phenotype had the highest similarity. As shown in Fig. 8, KO can be 


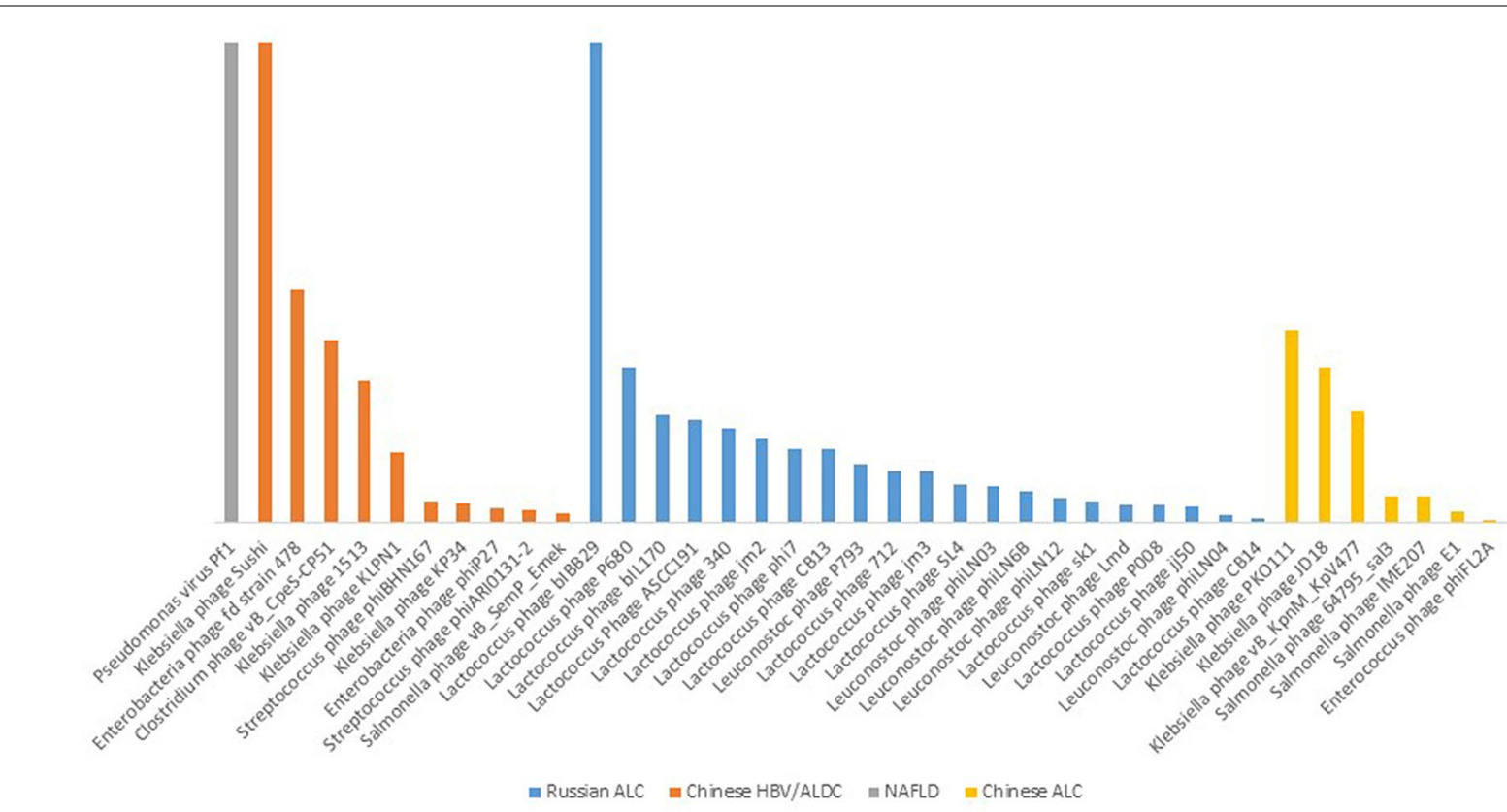

Fig. 6 Etiological-specific enteric bacteriophages among cirrhotic patients. Each bar plot indicates the frequency of a bacteriophage species detected in an individual etiology exhaustively

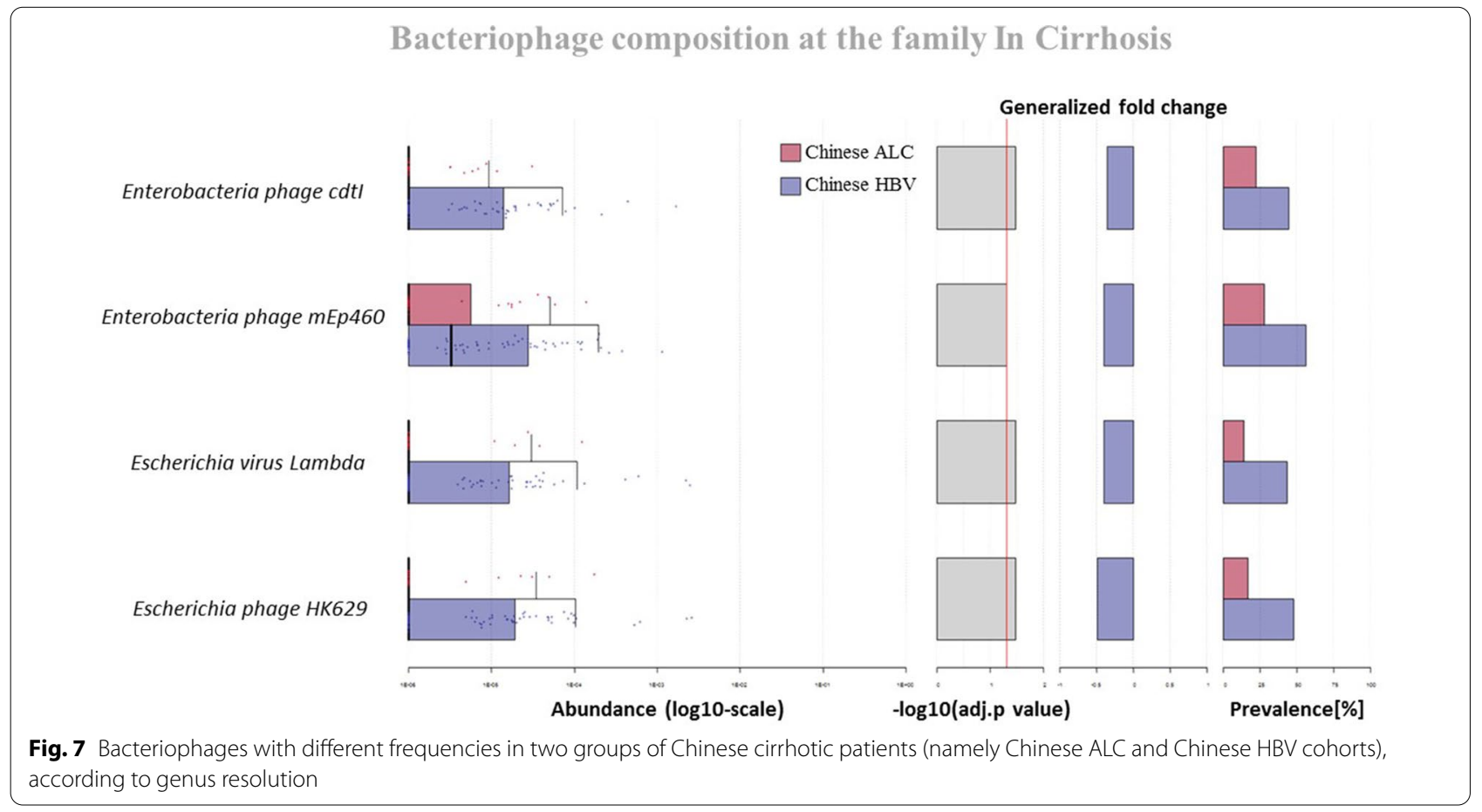

a proper discriminating feature compared to taxonomic and other genomic features in classifying different etiologies of liver cirrhosis. According to the PCA analysis, taxonomic features cannot be proper markers to classify different etiologies of liver disorders. However, the KO functional analysis of the gut phageome could be a practical approach to discriminate liver cirrhosis with various etiologies. Figure 9 shows the functional annotation of the phageome genes identified in the cirrhotic patients based on $\mathrm{KO}$ analysis. Carbohydrate active enzyme was 


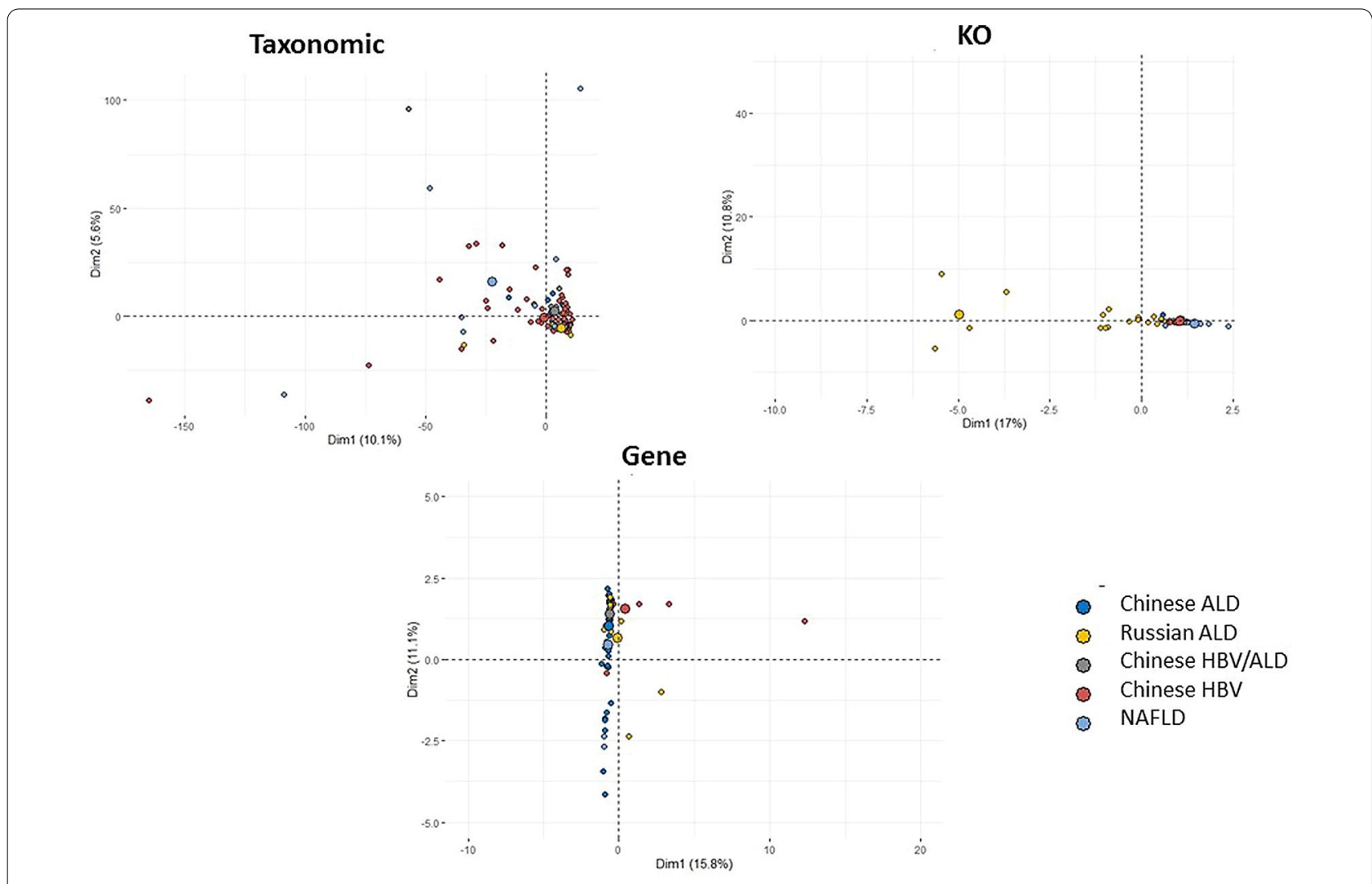

Fig. 8 PCA analysis of three resolutions, including taxonomic, gene, and $\mathrm{KO}$ analysis. As it was concluded, KO is a better discriminating factor two other features are compared

specific KEGG pathway in Chinese ALC group, while homoacetogenesis was unique pathway for Russian ALC casese. Moreover, transporters and saccharide synthesis pathway were spesefic KOs in NAFLD cohort. Nucleic acid metabolism and amino acid utilization biosynthesis metabolism were the common pathways identified in all of cirrhotic groups. Additional file 7: Figure S1 provide further information that revealed the results of FuncTree2 based on $\mathrm{KO}$ analysis in each cirrhotic cohort.

\section{Discussion}

Advances in next-generation sequencing and metagenomic analyses have facilitated the investigation of the relationship between the human gut microbiome with individuals' health and diseases. Although existing knowledge on the role of the gut microbiome in human health mainly comes from the analysis of diversity and the composition of the bacterial community, little is explored about those of phages [45]. To the best of our knowledge, this study was the first to explore the gut phageome of patients with liver diseases from a structural and functional perspective. Accordingly, sophisticated bioinformatic methods were adopted to characterize the phageome in fecal samples from cirrhotic patients with different etiologies in previously published studies. Moreover, we examined the total gut phageome of liver disorders at three levels, namely taxonomic classification, gene catalog construction, and $\mathrm{KO}$ analysis.

We analyzed gut phageome composition in cirrhotic patients with different etiologies using the custom method and detected no change in $\alpha$ - and $\beta$-diversities. This finding is in agreement with the findings in our previous study, indicating no evidence of a significant difference in bacterial community diversity. On the other hand, our comparative evaluation revealed some differences in the enteric phageome in different cohorts at the family level. This was significantly observed in Chinese ALC and NAFLD cohorts, in which the Retroviridae family was eliminated. Although this change was observed in the Chinese ALC cohort, no significant expansion of the taxonomic richness of Retroviridae phages was noticed in the Russian ALC samples. These findings are particularly interesting as the samples were collected from cirrhotic patients with a specific etiology (i.e., alcohol-induced cirrhosis) at two independent and geographically distinct regions. These findings would suggest geography, 


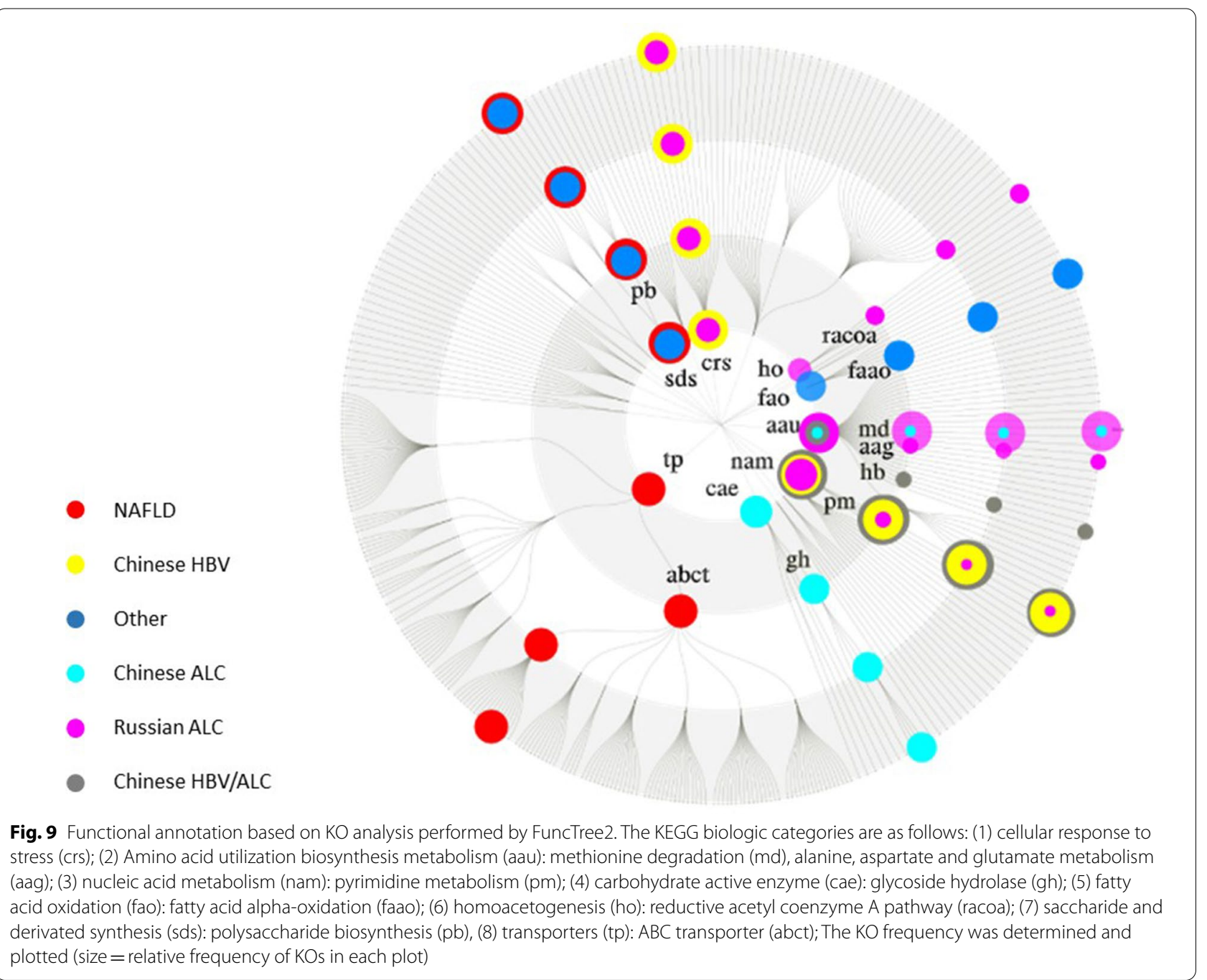

ethnicity, or lifestyle-based alterations in gut phageome communities. In a study by Pérez-Brocal et al. [46], phage strains from the family Retroviridae were more prevalent in IBD patients than in normal controls.

Interestingly, we found a phage signature in each etiology by determining individualized phage strains in each cohort. The comparison of the cohorts indicated that gut phageome could be unique at the strain level among cirrhotic patients concerning the etiology. For example, the results revealed that Pf1 phage belonging to the family Inoviridae was significantly overrepresented in NAFLD patients and that the other cohorts exhibited the total disappearance of this phage. Pf1 infects Pseudomonas aeruginosa strains and contributes to bacterial shortterm evolution and virulence [47]. In another example, we indicated that two phage species in the genus Lambdavirus (namely Escherichia virus Lambda and Escherichia phage HK629) were more prevalent in the Chines HBV than the Chines ALC cohorts. As described, the human gut microbiome is expected to be strongly affected by the activity of the phage composition [17]. Accordingly, the increased prevalence of certain phages could be accompanied by specific dynamic interactions with their bacterial and human hosts [48-50]. Therefore, considering its specific etiology, such phage overrepresentation detected in our analysis may impose some alterations in the gut bacterial community in cirrhosis. More importantly, these data could be applied to detect whether the gut phageome signature is predictive of the diagnosis of liver cirrhosis induced by a specific etiology. However, some data in the present study suggested geography, ethnicity, or lifestyle-based alterations in the gut phageome of the cohorts. For example, compared to the other cohorts, the Chines cirrhotic cohorts phageome, regardless of their etiology, was characterized with remarkably overrepresented certain Enterobacteriaceaespecific phage groups, including those specific to Klebsiella and Salmonella spp. Another significant finding of 
our analysis was the significant differences in the richness of the gut phageome between the Russian ALC and the Chines ALC patients. Although the etiology of these patients was alcoholic cirrhosis, the Russian ALC exhibited a specific expansion of Lactobacillus and Leuconostoc-specific phages. In contrast, the predominant phages in the Chines ALC cohort were Enterobacteriaceae-specific strains.

Given that the taxonomic resolution of the gut phageome in liver cirrhosis with different etiologies can be similar and that their functionality can differ in several etiologies, we aimed to investigate the gut phageome in liver cirrhosis using a novel and comprehensive gut gene catalog for the 139 cirrhotic patients from different etiologies. We found that the core phageome of cirrhotic patients consists of at least 21 phage species, suggesting that these phages could be used to characterize cirrhosis by the gut phageome composition. However, these data do not account for temporal dynamics among the gut phageome, intestinal bacterial community, and the host. Accordingly, the findings on these phage categories are conservative and may need to be retested with temporal data from more subjects. Among these 21 phages, the newly-identified uncultured crAssphage (NC_024711.1) was the predominant strain in cirrhotic patients. Moreover, this phage is also known to be a highly prevalent phage in the unknown sequences of human fecal metagenomes [51]. Bacterial species belonging to the phylum Bacteroidetes, commonly found in gut mucosal microbiota, were predicted to be the host of phage crAssphage [52].

To the best of our knowledge, a phage gene catalog of distinct etiologies of liver cirrhosis was developed in this study for the first time. Remarkably, due to the lack of phage reference genomes and the high complexity of whole-community metagenomic sequencing data, it is challenging to discriminate phage and bacterial sequences in metagenomic datasets. In this study, the three different strategies, including CRISPR-based analysis, scaffolds generating by FastViromeExplorer, and scaffold generating by homologous proteins were applied to overcome the complications of constructing the gut phage catalog from whole-community metagenomic sequencing data. The largest majority of the identified phage scaffolds by our analysis were assigned as unclassified phages. The second majority of the identified phage scaffold in the current catalog was taxonomically related to the Siphoviridae family.

According to the results, we suggest that the significant discriminating features should be increased by using the functional analysis. As illustrated in Fig. 8, the PCA analysis results at both gene and $\mathrm{KO}$ levels revealed that $\mathrm{KO}$ might be a more accurate feature to predict the etiology of liver disorders; hence, more precise treatment and disease management protocols need to be developed and prescribed. KO can be considered as a more suitable discriminating feature in classifying different etiologies of liver cirrhosis, compared to taxonomic and even genomic features. Considerably, "K00986" and "K06905" were common in all six different groups. In this regard, the former is related to RNAdirected DNA polymerase, and the latter is related to an uncharacterized protein. Remarkably, each cohort has a significant number of KOs, for example, the NAFLD group has 36 dedicated KOs. Accordingly, the $\mathrm{KO}$ analysis could help more deeply investigate the exact function of the gut phageome, particularly in liver cirrhosis with different etiologies.

Our deep understanding of the composition and function of the gut phaegome in patients with liver diseases compared to healthy individuals can help guide us to find a unique "fingerprint marking" that may play a role in inter-individual phenotypic variation in disease development, prognosis, progression, and even response to treatment. In addition, further investigation could open new insights into the potential therapeutic benefits of phages in liver diseases. In the recent decade, some clinical trials have investigated the safety and efficacy of phage therapy for gastrointestinal diseases. Phage-based therapy for the treatment of patients with alcoholic liver disease was suggested previously by Duan et al. [53]. However, further clinical trials with a larger cohort are to validate these novel therapeutic approaches for patients with alcoholic cirrhosis.

\section{Conclusions}

For decades, scientists have mainly disregarded the role of the gut microbiome as a potential modulator in the development and progression of gastrointestinal tract diseases, including liver diseases. In the current study, we found some specific taxonomic and functional alternations in the gut phageome among cirrhotic patients with distinct etiologies. Pseudomonas virus Pf1 phage and uncultured phage crAssphage have been eliminated in the NAFLD patients individually. However, our findings indicated that KEGG orthology analysis of the gut phageome can be a suitable discriminating feature compared to taxonomic and other genomic features in classifying different etiologies of liver cirrhosis In addition to focusing on the composition of phageome in liver diseases, investigating its functional features might also be insightful. 


\section{Supplementary Information}

The online version contains supplementary material available at https://doi. org/10.1186/s13099-022-00482-4.

Additional file 1. Scripts and Commands: All codes and scripts used in this study are available at https://github.com/Naseri1374/phageome-inliver-cirrhosis. All information about each group including filtered genes (with FDR above 0.01), GhostKOALA and BlastKOALA results, specific KEGGs, pfam, cog, and eggnog results, and specific metabolic pathways are presented.

Additional file 2. Scripts and Commands: All codes and scripts used in this study are available at https://github.com/Naseri1374/phageome-inliver-cirrhosis. All information about each group including filtered genes (with FDR above 0.01), GhostKOALA and BlastKOALA results, specific KEGGs, pfam, cog, and eggnog results, and specific metabolic pathways are presented.

Additional file 3. Scripts and Commands: All codes and scripts used in this study are available at https://github.com/Naseri1374/phageome-inliver-cirrhosis. All information about each group including filtered genes (with FDR above 0.01), GhostKOALA and BlastKOALA results, specific KEGGs, pfam, cog, and eggnog results, and specific metabolic pathways are presented.

Additional file 4. Scripts and Commands: All codes and scripts used in this study are available at https://github.com/Naseri1374/phageome-inliver-cirrhosis. All information about each group including filtered genes (with FDR above 0.01), GhostKOALA and BlastKOALA results, specific KEGGs, pfam, cog, and eggnog results, and specific metabolic pathways are presented

Additional file 5. Scripts and Commands: All codes and scripts used in this study are available at https://github.com/Naseri1374/phageome-inliver-cirrhosis. All information about each group including filtered genes (with FDR above 0.01), GhostKOALA and BlastKOALA results, specific KEGGs, pfam, cog, and eggnog results, and specific metabolic pathways are presented

Additional file 6. Venn diagram results of $\mathrm{KO}$ analysis are shown.

Additional file 7: Figure S1. Functional annotation based on $\mathrm{KO}$ analysis performed by FuncTree2 in cirrhotic patients with various etiologies. The KEGG biologic categories are as follows: (1) cellular response to stress (crs); (2) Amino acid utilization biosynthesis metabolism (aau): methionine degradation (md), alanine, aspartate and glutamate metabolism (aag); (3) nucleic acid metabolism (nam): pyrimidine metabolism (pm); (4) carbohydrate Active enzyme(cae): glycoside hydrolase(gh); (5) fatty acid oxidation (fao): fatty acid alpha-oxidation (faao); (6) homoacetogenesis (ho): reductive acetyl coenzyme A pathway(racoa); (7) saccharide and derivated synthesis (sds): polysaccharide biosynthesis(pb), (8) transporters(tp): ABC transporter(abct); The KO frequency was determined and plotted (size $=$ relative frequency of KOs in each plot).

\section{Acknowledgements}

The authors express special thanks to the members of the Foodborne and Waterborne Diseases Research Center, Research Institute for Gastroenterology and Liver Diseases, Shahid Beheshti University of Medical Sciences, Tehran, Iran.

\section{Authors' contributions}

$M N$, FP, and JZ designed the study. MN, FP, and JZ performed the analyses. $M N, J Z, H H$, and $A Y$ interpreted the data. MN, FP, HH, and HAA wrote the article with input from all authors. All authors read and approved the final manuscript.

\section{Funding}

None.

\section{Availability of data and materials}

All metagenomics datasets used in this study were obtained from the NCBI short read archive (SRA) database (PRJEB6337; PRJNA373901; and PRJEB18041) generated in three previously-published microbiome studies $[10,24,25]$.

\section{Declarations}

Ethics approval and consent to participate

No ethical approval was required for this study.

\section{Consent for publication}

Not applicable.

\section{Competing interests}

The authors declared no conflict of interests.

\section{Author details}

${ }^{1}$ Bioinformatics and Computational Omics Lab (BioCOOL), Department of Biophysics, Faculty of Biological Sciences, Tarbiat Modares University, Tehran, Iran. ${ }^{2}$ Laboratory of Complex Biological Systems and Bioinformatics (CBB), Institute of Biochemistry and Biophysics (IBB), University of Tehran, Tehran, Iran. ${ }^{3}$ Foodborne and Waterborne Diseases Research Center, Research Institute for Gastroenterology and Liver Diseases, Shahid Beheshti University of Medical Sciences, Shahid Arabi Ave., Yemen St., Velenjak, Tehran, Iran. ${ }^{4}$ Medical Imaging Center, Karaj, Alborz, Iran. ${ }^{5}$ Basic and Molecular Epidemiology of Gastrointestinal Disorders Research Center, Research Institute for Gastroenterology and Liver Diseases, Shahid Beheshti University of Medical Sciences, Tehran, Iran. ${ }^{6}$ Department of Neuroscience, University of California, San Diego, 9500 Gilman Drive, La Jolla, CA 92093-0662, USA.

Received: 10 October 2021 Accepted: 3 February 2022

Published online: 15 February 2022

References

1. Cani PD. Human gut microbiome: hopes, threats and promises. Gut. 2018;67(9):1716-25.

2. Lapidot Y, Amir A, Nosenko R, Uzan-Yulzari A, Veitsman E, Cohen-Ezra O, Davidov Y, Weiss P, Bradichevski T, Segev $S$, et al. Alterations in the gut microbiome in the progression of cirrhosis to hepatocellular carcinoma. mSystems. 2020;5(3):e00153-e1120.

3. Anand G, Zarrinpar A, Loomba R. Targeting dysbiosis for the treatment of liver disease. Semin Liver Dis. 2016;36(1):37-47.

4. Pasolli E, Truong DT, Malik F, Waldron L, Segata N. Machine learning metaanalysis of large metagenomic datasets: tools and biological insights. PLoS Comput Biol. 2016;12(7):e1004977.

5. Asrani SK, Devarbhavi H, Eaton J, Kamath PS. Burden of liver diseases in the world. J Hepatol. 2019;70(1):151-71.

6. Sarin SK, Choudhury A. Acute-on-chronic liver failure: terminology, mechanisms and management. Nat Rev Gastroenterol Hepatol. 2016;13(3):131-49.

7. Pinzani M, Rosselli M, Zuckermann M. Liver cirrhosis. Best Pract Res Clin Gastroenterol. 2011;25(2):281-90.

8. Acharya C, Bajaj JS. Altered microbiome in patients with cirrhosis and complications. Clin Gastroenterol Hepatol. 2019;17(2):307-21.

9. Oikonomou T, Papatheodoridis G, Samarkos M, Goulis I, Cholongitas E. Clinical impact of microbiome in patients with decompensated cirrhosis. World J Gastroenterol. 2018;24:3813-20.

10. Loomba R, Seguritan V, Li W, Long T, Klitgord N, Bhatt A, Dulai PS, Caussy C, Bettencourt R, Highlander SK, et al. Gut microbiome-based metagenomic signature for non-invasive detection of advanced fibrosis in human nonalcoholic fatty liver disease. Cell Metab. 2017;25(5):1054-1062.e1055.

11. Zhao Y, Zhou J, Liu J, Wang Z, Chen M, Zhou S. Metagenome of gut microbiota of children with nonalcoholic fatty liver disease. Front Pediatr. 2019;7:518-518. 
12. Wylie KM, Weinstock GM, Storch GA. Emerging view of the human virome. Transl Res J Lab Clin Med. 2012;160(4):283-90.

13. Paez-Espino D, Eloe-Fadrosh EA, Pavlopoulos GA, Thomas AD, Huntemann M, Mikhailova N, Rubin E, Ivanova NN, Kyrpides NC. Uncovering earth's virome. Nature. 2016:536(7617):425-30.

14. Norman Jason M, Handley Scott A, Baldridge Megan T, Droit L, Liu Catherine Y, Keller Brian C, Kambal A, Monaco Cynthia L, Zhao G, Fleshner $P$, et al. Disease-specific alterations in the enteric virome in inflammatory bowel disease. Cell. 2015;160(3):447-60.

15. Shkoporov AN, Hill C. Bacteriophages of the human gut: the "known unknown" of the microbiome. Cell Host Microbe. 2019:25(2):195-209.

16. d'Humières C, Touchon M, Dion S, Cury J, Ghozlane A, Garcia-Garcera M, Bouchier C, Ma L, Denamur E, Rocha EPC. A simple, reproducible and cost-effective procedure to analyse gut phageome: from phage isolation to bioinformatic approach. Sci Rep. 2019;9(1):11331.

17. Manrique P, Bolduc B, Walk ST, van der Oost J, de Vos WM, Young MJ. Healthy human gut phageome. Proc Natl Acad Sci USA. 2016;113(37):10400-5.

18. Hoyles L, McCartney AL, Neve H, Gibson GR, Sanderson JD, Heller KJ, van Sinderen D. Characterization of virus-like particles associated with the human faecal and caecal microbiota. Res Microbiol. 2014;165(10):803-12.

19. Sausset R, Petit MA, Gaboriau-Routhiau V, De Paepe M. New insights into intestinal phages. Mucosal Immunol. 2020;13(2):205-15.

20. Monaco Cynthia L, Gootenberg David B, Zhao G, Handley Scott A, Ghebremichael Musie S, Lim Efrem S, Lankowski A, Baldridge Megan T, Wilen Craig B, Flagg M, et al. Altered virome and bacterial microbiome in human immunodeficiency virus-associated acquired immunodeficiency syndrome. Cell Host Microbe. 2016;19(3):311-22.

21. Reyes A, Blanton LV, Cao S, Zhao G, Manary M, Trehan I, Smith MI, Wang D, Virgin HW, Rohwer F, et al. Gut DNA viromes of Malawian twins discordant for severe acute malnutrition. Proc Natl Acad Sci USA. 2015;112(38):11941-6

22. Ma Y, You X, Mai G, Tokuyasu T, Liu C. A human gut phage catalog correlates the gut phageome with type 2 diabetes. Microbiome. 2018;6(1):24.

23. Li J, Jia H, Cai X, Zhong H, Feng Q, Sunagawa S, Arumugam M, Kultima JR, Prifti $\mathrm{E}$, Nielsen T, et al. An integrated catalog of reference genes in the human gut microbiome. Nat Biotechnol. 2014;32(8):834-41.

24. Qin N, Yang F, Li A, Prifti E, Chen Y, Shao L, Guo J, Le Chatelier E, Yao J, Wu $L$, et al. Alterations of the human gut microbiome in liver cirrhosis. Nature. 2014;513(7516):59-64

25. Tyakht AV, Dubinkina VB, Odintsova VY, Yarygin KS, Kovarsky BA, Pavlenko AV, Ischenko DS, Popenko AS, Alexeev DG, Taraskina AY, et al. Data on gut metagenomes of the patients with alcoholic dependence syndrome and alcoholic liver cirrhosis. Data Brief. 2017;11:98-102.

26. Robinson MD, McCarthy DJ, Smyth GK. edgeR: a Bioconductor package for differential expression analysis of digital gene expression data. Bioinformatics. 2010;26(1):139-40.

27. Langmead B, Salzberg SL. Fast gapped-read alignment with Bowtie 2. Nat Methods. 2012;9(4):357-9.

28. Ritchie ME, Phipson B, Wu D, Hu Y, Law CW, Shi W, Smyth GK. limma powers differential expression analyses for RNA-sequencing and microarray studies. Nucleic Acids Res. 2015;43(7):e47-e47.

29. Tithi SS, Aylward FO, Jensen RV, Zhang L. FastViromeExplorer: a pipeline for virus and phage identification and abundance profiling in metagenomics data. PeerJ. 2018:6:e4227.

30. Li D, Liu C-M, Luo R, Sadakane K, Lam T-W. MEGAHIT: an ultra-fast singlenode solution for large and complex metagenomics assembly via succinct de Bruijn graph. Bioinformatics. 2015;31(10):1674-6.

31. Grissa I, Vergnaud G, Pourcel C. CRISPRFinder: a web tool to identify clustered regularly interspaced short palindromic repeats. Nucleic Acids Res. 2007;35(suppl_2):W52-7.

32. Buchfink $B$, Xie $C$, Huson DH. Fast and sensitive protein alignment using DIAMOND. Nat Methods. 2015;12(1):59-60.

33. Fu L, Niu B, Zhu Z, Wu S, Li W. CD-HIT: accelerated for clustering the next-generation sequencing data. Bioinformatics (Oxford, England). 2012:28(23):3150-2.

34. Dixon P. VEGAN, a package of $R$ functions for community ecology. J Veg Sci. 2003;14(6):927-30.

35. McMurdie PJ, Holmes S. phyloseq: an R package for reproducible interactive analysis and graphics of microbiome census data. PLoS ONE. 2013:8(4):e61217.
36. Wickham H. ggplot2. Wiley Interdiscip Rev Comput Stat. 2011;3(2):180-5.

37. Lee W-P, Stromberg MP, Ward A, Stewart C, Garrison EP, Marth GT. MOSAIK a hash-based algorithm for accurate next-generation sequencing shortread mapping. PLoS ONE. 2014;9(3):e90581.

38. Li H, Handsaker B, Wysoker A, Fennell T, Ruan J, Homer N, Marth G, Abecasis $G$, Durbin R. The sequence alignment/map format and SAMtools. Bioinformatics. 2009;25(16):2078-9.

39. Kanehisa M, Sato Y, Morishima K. BlastKOALA and GhostKOALA: KEGG tools for functional characterization of genome and metagenome sequences. J Mol Biol. 2016;428(4):726-31.

40. Tatusov RL, Fedorova ND, Jackson JD, Jacobs AR, Kiryutin B, Koonin EV, Krylov DM, Mazumder R, Mekhedov SL, Nikolskaya AN, et al. The COG database: an updated version includes eukaryotes. BMC Bioinform. 2003:4:41.

41. Huerta-Cepas J, Szklarczyk D, Forslund K, Cook H, Heller D, Walter MC, Rattei T, Mende DR, Sunagawa S, Kuhn M, et al. eggNOG 4.5: a hierarchical orthology framework with improved functional annotations for eukaryotic, prokaryotic and viral sequences. Nucleic Acids Res. 2016:44(D1):D286-93

42. Sonnhammer ELL, Eddy SR, Birney E, Bateman A, Durbin R. Pfam: Multiple sequence alignments and HMM-profiles of protein domains. Nucleic Acids Res. 1998;26(1):320-2.

43. Marchler-Bauer A, Panchenko AR, Shoemaker BA, Thiessen PA, Geer LY, Bryant SH. CDD: a database of conserved domain alignments with links to domain three-dimensional structure. Nucleic Acids Res. 2002;30(1):281-3

44. Uchiyama T, Irie M, Mori H, Kurokawa K, Yamada T. FuncTree: functional analysis and visualization for large-scale omics data. PLOS ONE. 2015;10(5):e0126967.

45. Tetz G, Brown SM, Hao Y, Tetz V. Parkinson's disease and bacteriophages as its overlooked contributors. Sci Rep. 2018;8(1):10812.

46. Pérez-Brocal V, García-López R, Nos P, Beltrán B, Moret I, Moya A. Metagenomic analysis of crohn's disease patients identifies changes in the virome and microbiome related to disease status and therapy, and detects potential interactions and biomarkers. Inflamm Bowel Dis. 2015:21(11):2515-32.

47. Knezevic P, Voet M, Lavigne R. Prevalence of Pf1-like (pro)phage genetic elements among Pseudomonas aeruginosa isolates. Virology. 2015;483:64-71.

48. Mirzaei MK, Maurice CF. Ménage à trois in the human gut: interactions between host, bacteria and phages. Nat Rev Microbiol. 2017:15(7):397-408

49. Manrique P, Dills M, Young MJ. The human gut phage community and its implications for health and disease. Viruses. 2017;9(6):141.

50. Hsu BB, Gibson TE, Yeliseyev V, Liu Q, Lyon L, Bry L, Silver PA, Gerber GK. Dynamic modulation of the gut microbiota and metabolome by bacteriophages in a mouse model. Cell Host Microbe. 2019;25(6):803-814.e805.

51. Reyes A, Haynes M, Hanson N, Angly FE, Heath AC, Rohwer F, Gordon I. Viruses in the faecal microbiota of monozygotic twins and their mothers. Nature. 2010;466(7304):334-8

52. Dutilh BE, Cassman N, McNair K, Sanchez SE, Silva GG, Boling L, Barr JJ, Speth DR, Seguritan V, Aziz RK, et al. A highly abundant bacteriophage discovered in the unknown sequences of human faecal metagenomes. Nat Commun. 2014;5:4498.

53. Duan $Y$, Llorente $C$, Lang $S$, Brandl $K$, Chu H, Jiang L, White RC. Bacteriophage targeting of gut bacterium attenuates alcoholic liver disease. Nature. 2019;575(7783):505-11

\section{Publisher's Note}

Springer Nature remains neutral with regard to jurisdictional claims in published maps and institutional affiliations. 